\title{
Widely-Spaced Comb-Like Polymers Having Fluoroalkyl Side Chains
}

\author{
Kenji YoKOTA and Tadamichi HIRABAYASHI \\ Materials Research Laboratory, Nagoya Institute of Technology, \\ Gokiso-cho, Showa-ku, Nagoya 466, Japan
}

(Received December 24, 1984)

\begin{abstract}
An alternating copolymer of butadiene and a fluoroalkyl methacrylate $\mathbf{I}_{\mathrm{f}}$ and its hydrogenated copolymer $\mathbf{I I}_{\mathrm{f}}$ were synthesized and identified. We designated these copolymers as widely-spaced comb-like polymers as we did the alkyl side chain copolymers in our previous paper [Polym. J., 15, 891 (1983)].
\end{abstract}

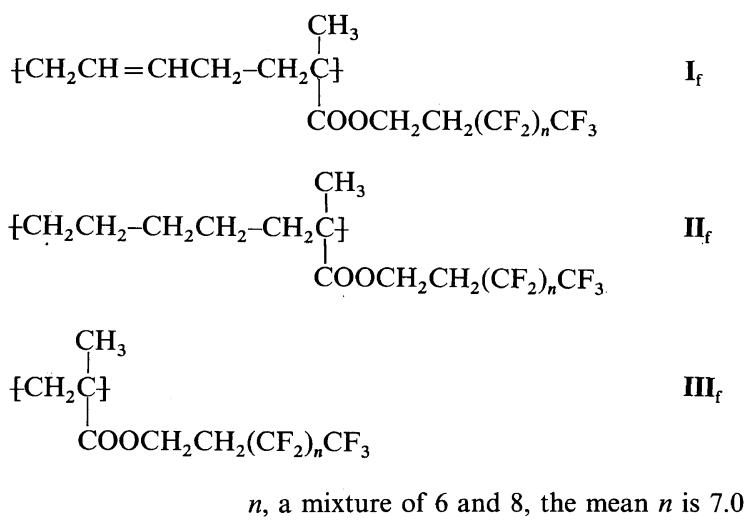

The fluoroalkyl methacrylate homopolymer $\mathbf{I I I}_{\mathrm{f}}$ is a brittle plastic but $\mathbf{I}_{\mathrm{f}}$ and $\mathbf{I I}_{\mathrm{f}}$ are extensible elastomers. Although $\mathbf{I I I}_{\mathrm{f}}$ is soluble in benzotrifluoride only when warmed, $\mathbf{I}_{\mathrm{f}}$ and $\mathbf{I I}_{\mathbf{f}}$ are soluble at room temperature. The cast films of $\mathbf{I}_{\mathrm{f}}, \mathbf{I I}_{\mathrm{f}}$, and $\mathbf{I I I}_{\mathrm{f}}$ showed the same contact angles of water or $n$ alkane droplets. Differential scanning calorimetry study demonstrated the crystallization of the fluoroalkyl side chains of $\mathbf{I}_{\mathbf{f}}$ and $\mathbf{I I}_{\mathbf{f}}$ in spite of their wide spaces. The fluoroalkyl side chains were found to be crystallizable more easily than the alkyl side chains of the same carbon atom numbers.

KEY WORDS Comb-Like Polymer / Widely-Spaced Comb-Like Polymer / Butadiene / Methacrylate / Fluoroalkyl Group / Alternating Copolymer / Hydrogenation / Contact Angle / Critical Surface Tension / Differential Scanning Calorimetry / Crystallization /

In our previous paper, ${ }^{1}$ alternating butadiene-long alkyl methacrylate copolymers I and their hydrogenated copolymers II were synthesized and identified.<smiles>[R]OC(=O)C(C)CCC=CC</smiles><smiles>[R]OC(=O)C(C)CCCCC</smiles>

$\mathrm{R}=-\left(\mathrm{CH}_{2}\right)_{n} \mathrm{H} n=12,14,16,18$

I In these copolymers, the long alkyl side chains appear on every six main chain carbon atoms. 
Therefore we designated them as widelyspaced comb-like polymers. In the conventional comb-like polymers, e.g., in the long alkyl methacrylate homopolymers III,<smiles>[R]OC(=O)C(C)C</smiles>

III

the side chains appear on every two main chain carbon atoms. Differential scanning calorimetry (DSC) and X-ray studies showed that the alkyl side chains of the widely-spaced comblike polymers could crystallize in the same order as those of the conventional comb-like polymers.

In the present paper, the synthesis and properties of such types of widely-spaced comb-like polymers having fluoroalkyl side chains are studied to confirm the above conclusion that also the long side chains of the widely-spaced comb-like polymers could crystallize. We also are interested in such polymers because of the peculiar physical properties of fluorocarbons and fluorinated polymers. The fluoroalkyl side chains can crystallize and do more easily than the alkyl side chains. The wetting property of these copolymers is also investigated.

\section{EXPERIMENTAL}

\section{Monomers}

The fluoroalkyl methacrylate monomer was prepared by the reaction of an excess of methyl methacrylate and a fluoroalkyl alcohol in the presence of $p$-toluenesulfonic acid and hydroquinone at reflux. The fluoroalkyl alcohol, $\mathrm{CF}_{3}\left(\mathrm{CF}_{2}\right)_{n} \mathrm{CH}_{2} \mathrm{CH}_{2} \mathrm{OH}$, a mixture of $n=6$ and 8 , was given by Daikin Industries Ltd. The monomer was purified by repeated fractional distillations. Yield was $60 \%$; bp $95.5-97.0^{\circ} \mathrm{C}$ [4 $\mathrm{mmHg}(0.5 \mathrm{kP})] .{ }^{1} \mathrm{H}$ nuclear magnetic resonance (NMR) spectrum at $200 \mathrm{MHz}$ in $\mathrm{CDCl}_{3}$ : $\delta 1.94 \mathrm{ppm}\left(\mathrm{t}, 3 \mathrm{H},-\mathrm{CH}_{3}\right), 2.52 \mathrm{ppm}(\mathrm{m}, 2 \mathrm{H}$ $\left.-\mathrm{CH}_{2}-\mathrm{CF}_{2}-\right), 4.47 \mathrm{ppm}\left(\mathrm{t}, 2 \mathrm{H},-\mathrm{OCH}_{2}-\right), 5.60$ and $6.15 \mathrm{ppm}\left(\mathrm{m}, 2 \mathrm{H}, \mathrm{CH}_{2}=\mathrm{C}-\right.$ ). Infrared (IR) spectrum of a liquid film: $v_{-\mathrm{C}=0} 1730 \mathrm{~cm}^{-1}$, $v_{-\mathrm{C}}=\mathrm{C}_{-} 1643 \mathrm{~cm}^{-1}, v_{\mathrm{C}-\mathrm{O}}$ around $1200 \mathrm{~cm}^{-1}$, and $\delta_{\text {olefinic CH }} 940 \mathrm{~cm}^{-1}$. Yield was $60 \%$. The mean composition $n$ of this monomer was determined by its $\mathrm{CH}_{3}$ peak intensity in an ${ }^{1} \mathrm{H}$ NMR spectrum by adding anisole as an internal standard and found to be 7.0. Extra pure butadiene was commercially obtained.

\section{Other Reagents}

Ethylaluminum sesquichloride from Ethyl Corp. was distilled under vacuum and dissolved in toluene $\left(3.89 \mathrm{~mol} \mathrm{dm}^{-3}\right)$. Extra pure vanadyl chloride was dissolved in hexane as received $\left(0.31 \mathrm{~mol} \mathrm{dm}^{-3}\right)$. Extra pure $p$-toluenesulfonylhydrazide was recrystallized from ethanol. Solvents were purified as usual.

\section{Polymerization}

In a nitrogen-purged $18^{\Phi} \times 150 \mathrm{~mm}$ test tube, the fluoroalkyl methacrylate $\left(3 \mathrm{~cm}^{3}\right.$, $9.3 \mathrm{mmol})$, toluene $\left(3 \mathrm{~cm}^{3}\right)$, and the ethylaluminum sesquichloride solution $\left(1 \mathrm{~cm}^{3}\right.$, $40 \mathrm{~mol} \%$ of methacrylate) were placed and mixed. Butadiene (ca. $2 \mathrm{~cm}^{3}, 22 \mathrm{mmol}$ ) was condensed at liquid nitrogen temperature. The content was mixed by shaking well on gradual warming. Finally the vanadyl chloride solution $\left(0.1 \mathrm{~cm}^{3}, 1 / 125 \mathrm{~mol}\right.$ of $\left.\mathrm{Al}\right)$ was introduced into the test tube. The test tube was sealed off and immersed in an ice-water bath overnight. A maroon colored mass precipitated. The content was poured into a large excess of methanol containing hydrochloric acid. Reprecipitations from the benzotrifluoride solution into methanol yielded white rubbery alternating butadiene-fluoroalkyl methacrylate copolymer $\mathbf{I}_{\mathrm{f}}$. It was dried in vacuum to a constant weight. Yield was $77 \% . \eta_{\mathrm{sp}} / c=0.68$ in benzotrifluoride $\left(c=0.5 \mathrm{~g} \mathrm{dm}^{-3}\right)$ at $30^{\circ} \mathrm{C}$.

The fluoroalkyl methacrylate homopolymer III $_{\mathrm{f}}$ was obtained from a mixture of the fluoroalkyl methacrylate $\left(3 \mathrm{~cm}^{3}\right)$, toluene $\left(3 \mathrm{~cm}^{3}\right)$, and $2,2^{\prime}$-azobisisobutyronitrile $(100 \mathrm{mg})$ by warming at $60^{\circ} \mathrm{C}$ for 1 day. It was reprecipi- 
tated twice from the benzotrifluoride solution into tetrahydrofuran and dried in vacuum. Yield was $88 \%$.

\section{Hydrogenation}

$\mathbf{I}_{\mathrm{f}}(1 \mathrm{~g})$ was dissolved in benzotrifluoride $\left(40 \mathrm{~cm}^{3}\right)$ with a trace of $t$-butylcatechol. The solution was stirred and heated with $p$ toluenesulfonylhydrazide ( $1.3 \mathrm{~g}, 4$ equivalents) at $100^{\circ} \mathrm{C}$ for $8 \mathrm{~h}$. The hydrogenated copolymer II $_{\mathrm{f}}$ was precipitated by pouring the solution into methanol and dried in vacuum. The yield was quantitative.

\section{Apparatus}

IR spectra were recorded for film samples on salt plates on a JASCO DS701G. ${ }^{1} \mathrm{H}$ $(200 \mathrm{MHz})$ and ${ }^{13} \mathrm{C}(50 \mathrm{MHz}) \mathrm{NMR}$ spectra were recorded for benzotrifluoride solutions on a Varian XL-200. Hexamethyldisiloxane (HMDS) was used as the internal reference. DSC was determined on a Rigaku-Denki Theremoflex provided with a low temperature unit. About a $20 \mathrm{mg}$ sample was taken in an aluminum pan covered with a lid. Both the heating and cooling rates were $5^{\circ} \mathrm{C} \mathrm{min}{ }^{-1}, \mathrm{X}$ Ray diffraction patterns were recorded for film samples on slide glasses using Ni-filtered $\mathrm{Cu}-\mathrm{K \alpha}$ radiation on a Rigaku-Denki RAD II diffractometer. The wetting property was determined on a Kyowa-Kagaku Contact Angle Meter CA-A for water and $n$-alkane droplets.

\section{RESULTS AND DISCUSSION}

\section{Polymer Structure}

The best evidence of the periodic structure of $\mathbf{I}_{\mathrm{f}}$ and $\mathbf{I I}_{\mathrm{f}}$ copolymers is given by the ${ }^{13} \mathrm{C}$ NMR spectra of $\mathbf{I I}_{\mathrm{f}}$ (Figures 1a and $\mathrm{b}$ ) as in the previous paper. Figure 1a shows eight peaks (two peaks at $29.56 \mathrm{ppm}$ are hardly observed to be split in this figure) besides the peaks due to the reference and the solvent. Assignment of

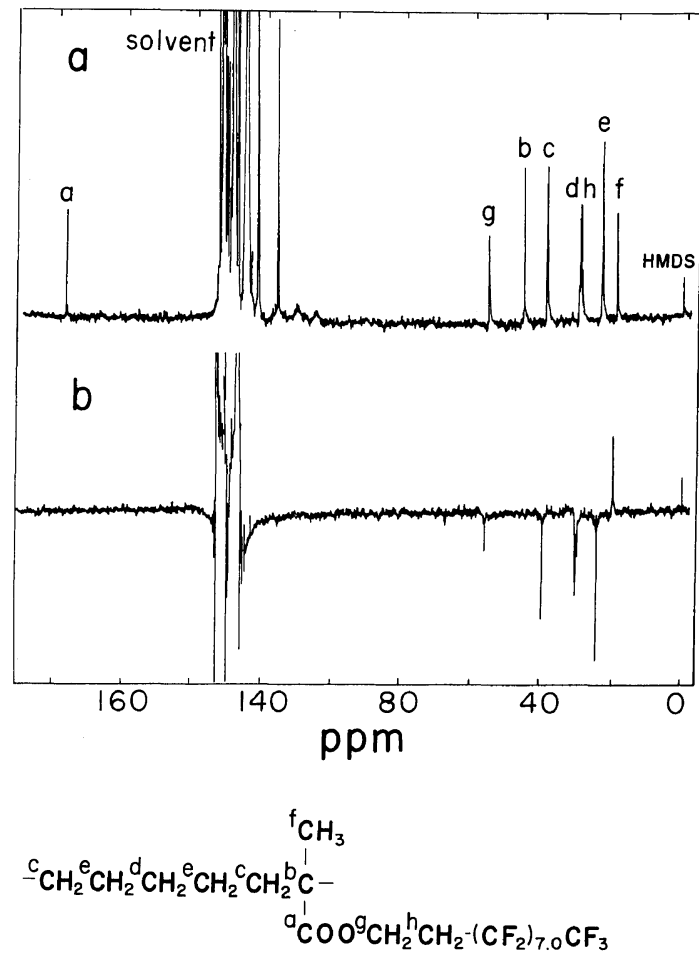

Figure 1. ${ }^{13} \mathrm{C}$ NMR spectra of $\mathbf{I I}_{\mathrm{f}}$ at $50 \mathrm{MHz}$ in benzotrifluoride at ambient temperature: a, proton noise decoupled; b, INEPT spectra.

these peaks to the eighteen carbons in one repeating unit was carried out by referring to 1) some standard chemical shift tables, 2) the spectra of the type II copolymers with various ester substituents previously reported, ${ }^{1}$ and 3 ) the INEPT (Insensitive Nuclear Enhancement through Polarization Transfer ${ }^{2}$ ) spectrum in the Figure 1b. Fortunately, the disappearance of the absorptions of fluorine-substituted carbons in the noise*1 and the structural symmetry about the quarternary carbon atom of the methacrylate unit simplified the spectrum. The assignment is shown in the figure. The ${ }^{\mathrm{h}} \mathrm{CH}_{2}$ absorption is split by the adjacent $\mathrm{CF}_{2}$. No other absorptions are observed and therefore the periodic structural purity of $\mathbf{I I}_{\mathrm{f}}$ is very

*1 Weak and complicated split peaks was observed between 100 and $125 \mathrm{ppm}$ for the fluorine-substituted carbons of the monomer in its ${ }^{13} \mathrm{C}$ NMR spectrum. 


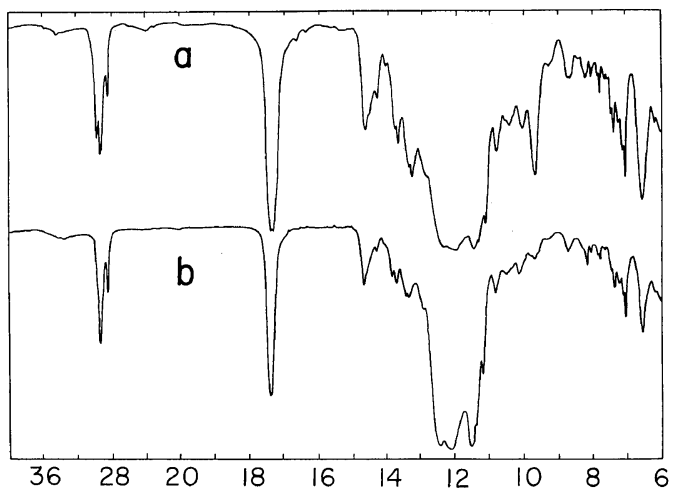

Figure 2. IR spectra of a) $\mathbf{I}_{f}$ and b) $\mathbf{I I}_{f}$ films.

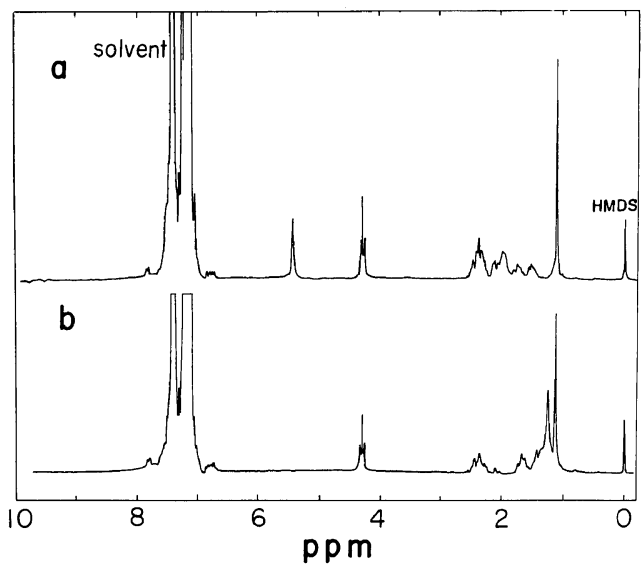

Figure 3. ${ }^{1} \mathbf{H}$ NMR spectra of a) $\mathbf{I}_{f}$ and b) $\mathbf{I I}_{f}$ at $200 \mathrm{MHz}$ in benzotrifluoride at $80^{\circ} \mathrm{C}$.

high. IR and ${ }^{1} \mathrm{H}$ NMR spectra of the $\mathbf{I}_{\mathrm{f}}$ and $\mathbf{I I}_{\mathrm{f}}$ copolymers are shown in Figures 2 and 3. By these figures, especially by the disappearance of olefinic proton absorption in Figure $3 b$, the hydrogenation of $\mathbf{I}_{\mathrm{f}}$ to $\mathbf{I I}_{\mathrm{f}}$ seems complete. Thus, we have two types of widely-spaced comb-like polymers having fluoroalkyl side chains.

\section{General Properties of Copolymers}

While the homopolymer III $_{\mathbf{f}}$ is brittle, the copolymers $\mathbf{I}_{\mathrm{f}}$ and $\mathbf{I I}_{\mathrm{f}}$ are soft and extensible. Such a difference in material property was already observed for the polymers with various ester substituents (methyl ${ }^{3}$, ethyl, isopropyl, phenyl, and benzyl $\left.{ }^{4}\right)$. Type III homo-

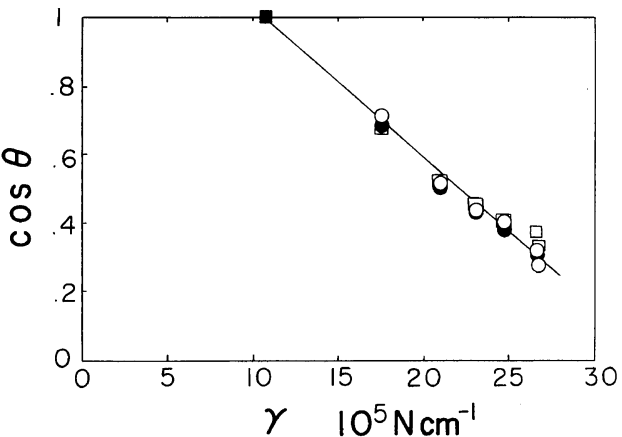

Figure 4. Zisman type plot for $\mathbf{I}_{\mathrm{f}}(\bigcirc), \mathbf{I I}_{\mathrm{f}}(\mathbf{O})$, and $\mathbf{I I I}_{\mathrm{f}}$ ( $\square)$. Abscissa: surface tension of $n$-alkanes, ordinate: cosine of contact angle. ( $\square$ ) indicates the literature value of a poly(fluoroalkyl methacrylate). ${ }^{6}$

Table I. Contact angles $\left({ }^{\circ}\right)$ of water and $n$-alkanes on $\mathbf{I}_{\mathrm{f}}, \mathbf{I I}_{\mathrm{f}}$, and $\mathbf{I I I}_{\mathrm{f}}$ films

\begin{tabular}{lrrr}
\hline Liquid & \multicolumn{1}{c}{$\mathbf{I}_{\mathbf{f}}$} & \multicolumn{1}{c}{$\mathbf{I I}_{\mathbf{f}}$} & III $_{\mathbf{f}}$ \\
\hline Water & 117.8 & 119.2 & 120.2 \\
Hexadecane & 73.8 & 73.3 & 70.1 \\
Tetradecane & 71.2 & 72.1 & 68.0 \\
Dodecane & 65.9 & 67.5 & 65.9 \\
Decane & 63.9 & 64.4 & 62.5 \\
Octane & 58.7 & 59.7 & 58.5 \\
Hexane & 44.3 & 46.8 & 47.9 \\
\hline
\end{tabular}

polymers were hard and brittle plastics and type I and II copolymers were highly extensible elastomers.

While III $_{\mathrm{f}}$ is soluble in benzotrifluoride only when warmed, $\mathbf{I}_{\mathbf{f}}$ and $\mathbf{I I}_{\mathrm{f}}$ are soluble at room temperature.

\section{Wetting Property}

During the reprecipitation experiment, we observed that III $_{\mathrm{f}}$ was stickly to a Teflon piece but $\mathbf{I}_{\mathrm{f}}$ and $\mathbf{I I}_{\mathrm{f}}$ were not. Here again we observed the influence of intervention of a butadiene unit or two ethylene units into the fluoroalkyl methacrylate sequence. The contact angle measurement of water and $n$-alkane droplets on these polymer films did not show appreciable differences.

Contact angles of water and a series of $n$ alkanes such as hexane, octane, decane, dodecane, tetradecane, and hexadecane on the 
films of the three polymers are shown in Table I and Figure 4. The film was cast on a slideglass from a polymer solution in benzotrifluoride. The slide-glass was set on the measuring stage of the apparatus and a droplet of the liquid was carefully placed on the film by a syringe. A photograph of the droplet profile on the film was taken and the contact angle was schematically determined on the photograph. The average value of six experiments was taken. As Table I and Figure 4 show, $\mathbf{I}_{\mathrm{f}}, \mathbf{I I}_{\mathrm{f}}$, and III $_{f}$ have the same contact angle for a given liquid within the experimental error $\left( \pm 2-3^{\circ}\right)$ and are water and oil repellent. A Zisman type plot ${ }^{5}$ is shown in Figure 4 where the extrapolation of surface tensions of the liquids $\gamma$ to $\cos \theta=1$, i.e., the critical surface tension of the polymers $\gamma_{c}$, coincides with the literature value $\left(10.6 \times 10^{-5} \mathrm{~N} \mathrm{~cm}^{-1}\right)$ of a poly(fluoroalkyl methacrylate). ${ }^{6}$ At the airsides of polymer films, the fluoroalkyl groups of the three polymers should be arranged on the surface to make surfaces similar in their wetting property.

\section{DSC Study}

In the heating mode DSC runs, while III $_{\mathrm{f}}$ constantly showed a sharp endothermic peak at $90^{\circ} \mathrm{C}, \mathbf{I}_{\mathrm{f}}$ and $\mathbf{I I}_{\mathrm{f}}$ showed poorly reproducible broad peaks around $40^{\circ} \mathrm{C}$. Therefore, as for the alkyl polymers in the previous paper, DSC traces of the cooling mode runs are shown in Figure 5 and subjected to discussion. In Figure $5, \mathbf{I}_{\mathrm{f}}$ and $\mathbf{I I}_{\mathrm{f}}$ show sharp peaks at $8^{\circ} \mathrm{C}$ and III $_{\mathrm{f}}$ at $84^{\circ} \mathrm{C}$. These exothermic peaks should be ascribed to the crystallization of the fluoroalkyl side chains of these widely-spaced and conventional comb-like polymers as observed for the alkyl polymers. Pittman ${ }^{7}$ reported the DSC study on the side chain crystallization of some poly(fluoroalkyl acrylates).

When we compare the DSC traces under identical experimental conditions of the present fluoroalkyl $\left(C_{10}\right)$ polymers with those of the alkyl (no data for $\mathrm{C}_{10}$, the smallest in the previous paper is $\mathrm{C}_{12}$ ) polymers, we can ob-

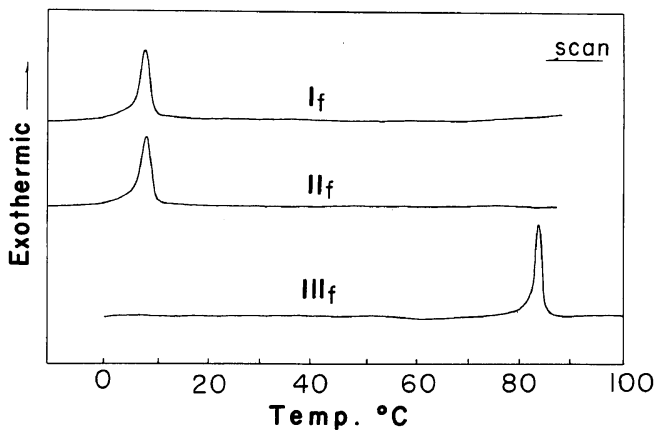

Figure 5. DSC traces of $\mathbf{I}_{f}, \mathbf{I I}_{f}$, and $\mathbf{I I I}_{f}$ determined on cooling mode.

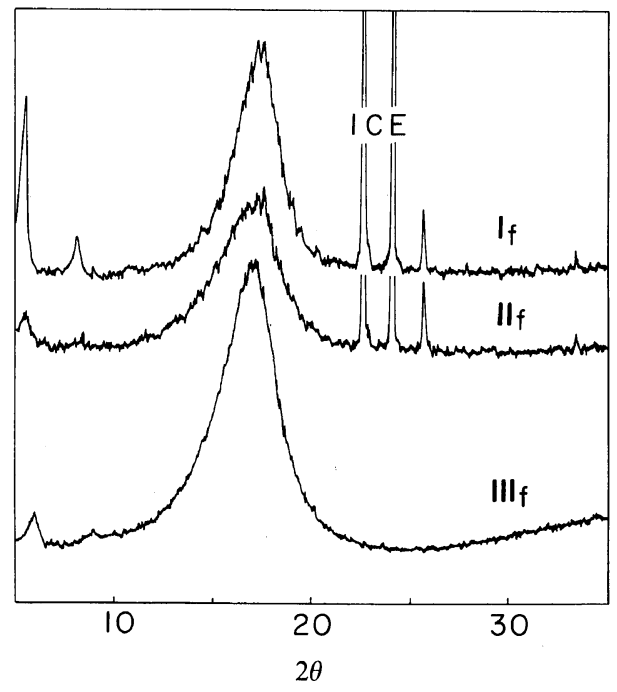

Figure 6. X-Ray diffraction patterns of $\mathbf{I}_{f}, \mathbf{I I}_{f}$, and $\mathbf{I I I}_{\mathrm{f}}$.

serve sharp peaks for the former in Figure 5 but only weak and obscure peaks for the latter in Figure 6 of the previous paper. Thus, the fluoroalkyl side chains can crystallize more easily than the alkyl side chains. The average numbers of crystallizing $\mathrm{CF}_{2}$ were tentatively estimated from the peak areas and the latent heat of fusion of polytetrafluoroethylene $\left(57.3 \mathrm{~kJ} \mathrm{~kg}^{-18}=700 \mathrm{cal}-\mathrm{CF}_{2}-^{-1}\right)$ and found to be about 1 for the three polymers as given in Table II. Our previous conclusion that the side chains of widely-spaced comb-like polymers can crystallize to the same extent to which those of conventional ones can is thus again 
Table II. Crystallizing temperature and number of crystallizing $\mathrm{CF}_{2}$

\begin{tabular}{lllc}
\hline & $\mathbf{I}_{\mathrm{f}}$ & $\mathbf{I I}_{\mathrm{f}}$ & III $_{\mathrm{f}}$ \\
\hline Crystallizing temp $/{ }^{\circ} \mathrm{C}$ & 8 & 8 & 84 \\
$\begin{array}{c}\text { Number of crystallizing } \\
\mathrm{CF}_{2}\end{array}$ & 0.9 & 1.1 & 1.1 \\
\hline
\end{tabular}

confirmed.

The lower temperature of crystallization for widely-spaced comb-like polymers than that for conventional one was explained in the previous paper in terms of the larger entropy of melting of widely-spaced ones due to their wider spaces.

\section{$X$-Ray Study}

Before recording the X-ray diffraction pattern of $\mathbf{I}_{\mathrm{f}}$ or $\mathbf{I I}_{\mathrm{f}}$, the sample slide glass was kept in a refrigerator and scanning was done while it was cold. Besides peaks due to ice, we can observe in Figure 6 peaks at $2 \theta=17^{\circ}$, the same

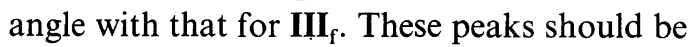
assigned to the crystallizing fluoroalkyl side chains on the analogy of the patterns of alkyl polymers.
Acknowledgment. The authors wish to thank Prof. Yuya Yamashita and Dr. Yasuhisa Tsukahara of Nagoya University for helping us to obtain the fluoroalkyl alcohol and assisting with the contact angle measurement. The partial support by a Grant-in-Aid for Scientific Research from the Ministry of Education, Science, and Culture of Japan is also acknowledged.

\section{REFERENCES}

1. K. Yokota, T. Kougo, and T. Hirabayashi, Polym. J., 15, 891 (1983).

2. G. A. Morris and R. Freeman, J. Am. Chem. Soc., 101, 761 (1979).

3. K. Yokota and T. Hirabayashi, Macromolecules, 14, 1613 (1981).

4. K. Yokota, T. Kougo, and T. Hirabayashi, Polym. J., 15, 349 (1983).

5. W. A. Zisman in "Advance in Chemistry," No. 34, American Chemical Society, Washington, D.C., 1964, p 1.

6. M. K. Bemett and W. A. Zisman, J. Phys. Chem., 66, 1207 (1962).

7. A. G. Pittman and B. A. Ludwig, J. Polym. Sic., A-1, 7, 3053 (1969).

8. J. Brandrup and E. H. Immergut, Ed., "Polymer Handbook," 2nd ed, Wiley-Interscience, New York, N. Y., 1975, p V-32. 